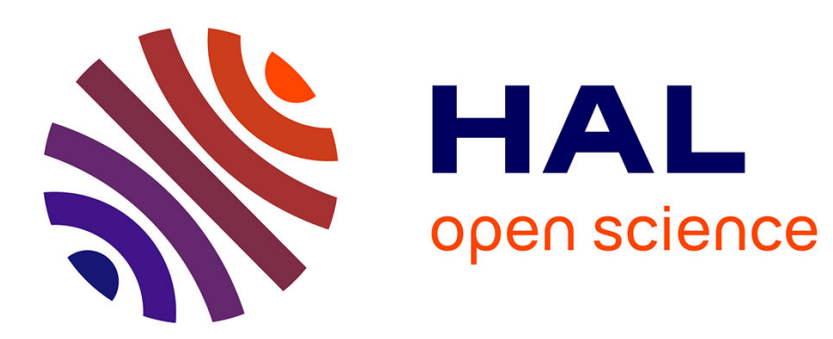

\title{
A flow-control policy minimizing the inventory cost in non deterministic environment
}

C. Libosvar, Jean-Marie Proth, Ph. Varin

\section{To cite this version:}

C. Libosvar, Jean-Marie Proth, Ph. Varin. A flow-control policy minimizing the inventory cost in non deterministic environment. RR-0551, INRIA. 1986. inria-00076003

\section{HAL Id: inria-00076003 \\ https://hal.inria.fr/inria-00076003}

Submitted on 24 May 2006

HAL is a multi-disciplinary open access archive for the deposit and dissemination of scientific research documents, whether they are published or not. The documents may come from teaching and research institutions in France or abroad, or from public or private research centers.
L'archive ouverte pluridisciplinaire HAL, est destinée au dépôt et à la diffusion de documents scientifiques de niveau recherche, publiés ou non, émanant des établissements d'enseignement et de recherche français ou étrangers, des laboratoires publics ou privés. 


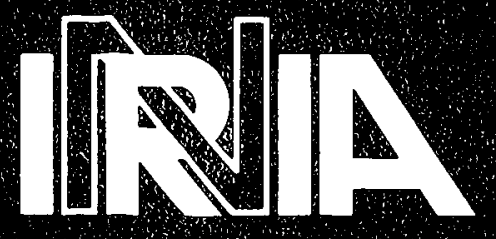

CENTRE DE ROCOUENCOURT

\section{Rapports de Recherche}

$$
\mathrm{N}^{\circ} 551
$$

\section{A FLOW-CONTROL POLICY MINIMIZING THE INVENTORY COST IN NON DETERMINISTIC ENVIRONMENT}

Institut National de Recherche en informatique et en Automatique

Dêmanine a voluceaut Doceuencourt

\section{4} 0000

Camille LIBOSVAR Jean-Marie PROTH Philippe VARIN 
March, 1986

\title{
A FLOW-CONTROL POLICY MINIMIZING THE INVENTORY COST IN NON DETERMINISTIC ENVIRONMENT
}

\author{
by \\ Camille LIBOSVAR * \\ Jean Marie PROTH ** \\ Philippo VARIN*
}

\begin{abstract}
The results developped in this paper present a flow-control policy in non deterministic environment : replenishment levels are computed in order to bound to a given epsilon ( control - parameter of the model) the probability of going out of stock, whereas the demand is supposed to be the accomplishment of a random normal variable. It is then shown that these replenishments are normal too and that it is possible to adapt this policy to any flow-shop system so long as it has been previously modelized as net of "tools" and buffers. In that case, we show that it is possible to choose the control-parameters assigned to each "tool" in order to minimise the global inventory cost.
\end{abstract}

keywords : production management, flow control, flow shop systems.

- Aluval Pechiney, 38340 zone industrielle de Voreppe.

** INRIA Nancy, Chateau du Montet 54500 Vandoeuvre. 


\section{INTRODUCTION :}

The management of a whole manufacturing system is a complex task for two main reasons : the existence of antagonistic evaluation criterions and the enormous amount of data to compute .

A solution consists in dividing this task in a multiple level hierarchy so that each level can be assigned a planning horizon consistent with the "resolution" of the image of the system that is required to take decisions over this horizon (e.g. long term decisions do not require a precise description of the production system : the global capacity and costs and some forecastings of the market trends are enough to take strategic decisions ). It is then obvious that the higher levels will have to deal with the long term and their decisions will induce the control constraints for the optimization at the lower levels. Each hierarchical level will then be assigned a set of criterions to optimize, these criterions being chosen in order that the effects of a decision taken at a given level to improve a criterion could be observed inside the horizon corresponding to this level.

Five different levels are commonly distinguished in a hierarchical management : strategy, flow control, planning, scheduling and real time control. This paper proposes a flow control method based on the replenishment control, in a non deterministic environment. The results are given for a two-stage system with infinite capacities but have been applied to a general flow shop system. 


\section{1/ DESCRIPTION OF THE REPLENISHMENT POLICY :}

The mathematical models developped in this paper assume that the production system to be managed has to meet a series of random-normal demands represented by their estimates ( mean values and standard deviations). Hence the problem : how to choose the job orders levels so that the probability of going out of stock in the future be reduced to a given "epsilon" and, at the same time, keep the minimal inventory level?

1) The kernel model:

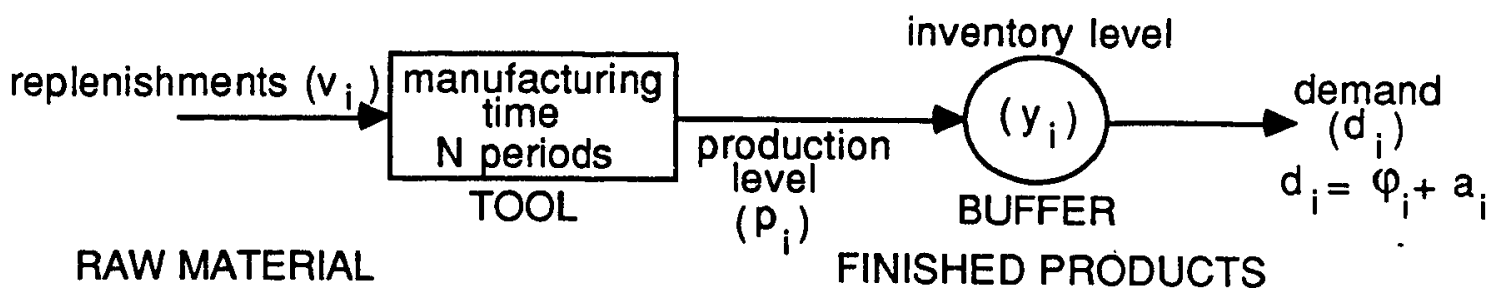

figure 1

The planification horizon is split in $\mathrm{h}$ periods (days, weeks ..)

The margin $a_{i}$ between the demands estimate for the $i$ th period and the actual demand $d_{j}$ is supposed to be a random-normal variable of null mean value and given standard deviation $\sigma_{i}$; two different margins $a_{i}$ and $a_{i}$ are supposed to be independent.

.No problems of maximal capacity are taken into account. (Neither is the fact that demands or replenishments could be negative according to the formulae which define them : these opportunities are supposed to be seldom enough ).

.Each replenishment $v_{i}$ is decided at the beginning of the $i$ th period so that the corresponding products become available at the beginning of the $(i+N)$ th period whereas the inventory level is measured at end of each period.

We then have the formulae :

$$
p_{i}=v_{i-N} \text { and } y_{i+1}=y_{i}+p_{i+1}-d_{i+1}
$$

The first one means that there is no throw-out or that raw material and finished product volumes are measured in different unities in order to take into account a mean throw-out rate.

The second one will be called "state-equation" and written :

$$
y_{i+1}=y_{i}+v_{i+1-N}-\left(\varphi_{i+1}+a_{i+1}\right)
$$

At the beginning of the first priod, we assume that we know :

the inventory level registered at the end of previous period : $y_{0}$

the last $N$ replenishments decided : $v_{1-N} \cdots . . v_{0}$ ( the corresponding

productions have not been completed yet ). ( standard deviations ).

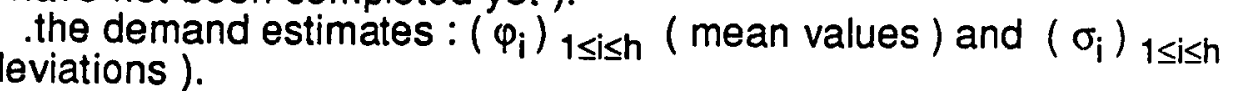


We have to fix the level of the replenishment $v_{1}$ so that $\operatorname{Pr}\left(y_{1+N} \leq 0\right)<\varepsilon$ : The so-called state equation gives us the means to link $y_{1+N}$ and $v_{1}$ :

$$
\begin{aligned}
y_{1+N} & =y_{N}+v_{1}-d_{1+N} \\
& =y_{0}^{*}+\sum_{i=1-N}^{0} v_{i}^{*}+v_{1}-\sum_{i=1}^{N+1} \varphi_{i}^{*}-\sum_{i=1}^{N+1} a_{i}
\end{aligned}
$$

The star symbol means that the referenced variables are known at the decision time; $\Sigma a_{i}$ is the sum of $N+1$ indepent random-normal variables hence a random normal variable too, of standard deviation $\Sigma_{1}=\sqrt{\sigma_{1}{ }^{2}+\sigma_{2}{ }^{2}+\ldots+\sigma_{N+1}}{ }^{2}$. Then, once $v_{1}$ is fixed, $y_{1+N}$ will be a random normal variable :

$$
\begin{aligned}
\operatorname{Pr}\left(y_{1+N} \leq 0\right)= & \operatorname{Pr}\left(\sum_{i=1}^{N+1} a_{i} \geq v_{1}+\left\{y_{0}^{*}+\sum_{i=1-N}^{0} v_{i}^{*}-\sum_{i=1}^{N+1} \varphi_{i}^{*}\right\}\right) \\
= & 1-F_{1}\left(v_{1}+\left\{y_{0}^{*}+\sum_{i=1-N}^{0} v_{i}^{*}-\sum_{i=1}^{N+1} \varphi_{i}^{*}\right\}\right) \\
& \text { where } F_{1} \text { is the distribution of } \sum_{i=1}^{N+1} a_{i}
\end{aligned}
$$

Then, $\operatorname{Pr}\left(y_{1+N} \leq 0\right)<\varepsilon$ is equivalent to :

$$
v_{1} \geq F_{1}^{-1}(1-\varepsilon)-\left\{y_{0}^{*}+\sum_{i=1-N}^{0} v_{i}^{*}-\sum_{i=1}^{N+1} \varphi_{i}^{*}\right\}
$$

If $F$ designates the distribution of a normal random variable of mean value 0 and standard deviation 1 , then $F_{1}(u)=F\left(u / \Sigma_{1}\right)$ and we will fix the first replenishment at the value given by the formula :

$$
v_{1}=\Sigma_{1} F^{-1}(1-\varepsilon)+\sum_{i=1}^{1+N} \varphi_{i}-\sum_{i=1-N}^{0} v_{i}-y_{0}
$$

The inventory level at the end of the $(\mathrm{N}+1)^{\text {th }}$ period will then be :

$$
\begin{aligned}
& y_{1+N}=y_{0}+\sum_{i=1-N}^{0} v_{i}-\sum_{i=1}^{1+N}\left(\varphi_{i}+a_{i}\right)+v_{1} \\
& y_{1+N}=\Sigma_{1} F^{-1}(1-\varepsilon) \cdot \sum_{i=1}^{1+N} a_{i}
\end{aligned}
$$


As these results depend only on the state equation and the hypothesis we have done concerning the demands, they can straight be extended to the successive replenishments : at the beginning of the $k^{\text {th }}$ period, we can forecast that the inventory level $y_{k+N}$ will be :

$$
\begin{aligned}
y_{k+N} & =y_{0}+\sum_{i=1}^{k+N} v_{i-N}-\sum_{i=1}^{k+N} d_{i} \\
& =\left\{y_{0}^{*}+\sum_{i=1-N}^{k-1} v_{i}^{*}-\sum_{i=1}^{k+N} \varphi_{i}^{*}-\sum_{i=1}^{k-1} a_{i}^{*}\right\}+v_{k}-\sum_{i=k}^{k+N} a_{i}
\end{aligned}
$$

$\mathrm{k}+\mathrm{N}$

where $\sum_{i=k}^{k+N} a_{i}$ is a normal random variable of mean value 0 and standard deviation equal to $\sum_{k}=\sqrt{\sigma_{k}^{2}+\sigma_{k+1}{ }^{2}+\ldots+\sigma_{k+N}{ }^{2}}$.

Then, $\operatorname{Pr}\left(y_{k+N} \leq 0\right)<\varepsilon$ is equivalent to :

$$
\operatorname{Pr}\left(\sum_{i=k}^{k+N} a_{i}<v_{k}+\left\{y_{0}^{*}+\sum_{i=1-N}^{k-1} v_{i}^{*}-\sum_{i=1}^{k+N} \varphi_{i}^{*}-\sum_{i=1}^{k-1} a_{i}^{*}\right\}\right) \geq 1-\varepsilon
$$

Hence the value to give to $v_{k}$ :

$$
v_{k}=\sum_{k} F^{-1}(1-\varepsilon)+\sum_{i=1}^{k+N} \varphi_{i}^{*}+\sum_{i=1}^{k-1} a_{i}^{*}-y_{0}^{*}-\sum_{i=1-N}^{k-1} v_{i}^{*}
$$

If the previous replenishment $v_{k-1}$ has been determined by means of the same formula, we remark that this one becomes shorter :

$$
v_{k}=F^{-1}(1-\varepsilon)\left(\Sigma_{k}-\Sigma_{k-1}\right)+\varphi_{k+N}+a_{k-1}^{*}
$$

In that case, we notice that the replenishment is the sum of three terms that we can analyse as :

$\rightarrow$ one term corresponding to an evolution of the reliability of the estimates with regard to the previous decision : if the standard deviation of the margin between demand and estimates is supposed to be constant, then this term is null.

$\rightarrow$ one term corresponding to the estimate for the period at the beginning of which the reordered quantity $v_{k}$ will be available for sell.

$\rightarrow$ and, at last, a term that is, in fact, the margin between demands and estimates registered during the previous period ! That means that the replenishments are equal to the estimates adjusted of the last difference fed back. 
And the inventory level at the end of the $(k+N)^{\text {th }}$ period will be :

$$
\begin{aligned}
& y_{k+N}=y_{0}^{*}+\sum_{i=1-N}^{k-1} v_{i}^{*}-\sum_{i=1}^{k+N} \varphi_{i}^{*}-\left\{\sum_{i=1}^{k-1} a_{i}^{*}+\sum_{i=k}^{k+N} a_{i}\right\}+v_{k} \\
& y_{k+N}=\Sigma_{k} F^{-1}(1-\varepsilon)-\sum_{i=k}^{k+N} a_{i}
\end{aligned}
$$

As the margins $a_{i}$ are supposed to have a null mean value ( non biased estimates ), this inventory level will be statistically equal to $\Sigma_{k} F^{-1}(1-\varepsilon)$ which depends only on the quality of the estimates (measured by the standard deviations) and the allowed risk, measured by the parameter $\varepsilon$.

\section{2) The two-levels system:}

We shall now consider the system consisting of two kernel models in series as shown on figure 2 : the demands which the upper part of the system has to meet are the replenishments decided by the lower part whereas the lower part has to meet a final random normal demand.

It is then obvious that the lower part of the system will work exactly as a single kernel model system ; that means if we assign it the control parameter $\varepsilon^{\prime}$, then the replenishment levels to be decided will be :

$$
\begin{array}{r}
v_{1}^{\prime}=\Sigma_{1}^{\prime} F^{-1}\left(1-\varepsilon^{\prime}\right)+\sum_{i=1}^{1+N^{\prime}} \varphi_{i}^{\prime}-\sum_{i=1-N}^{0} v_{i}^{\prime^{*}}-y_{0}^{\prime} \\
\forall k \geq 2 \quad v_{k}^{\prime}=F^{-1}\left(1-\varepsilon^{\prime}\right)\left(\Sigma_{k}^{\prime}-\Sigma_{k-1}^{\prime}\right)+\varphi_{k+N^{\prime}+a_{k-1}^{\prime}}^{\prime} \\
\text { where } \Sigma_{k}^{\prime}=\sqrt{\sum_{i=k}^{k+N_{i}^{\prime} \sigma_{i}^{\prime 2}}}
\end{array}
$$




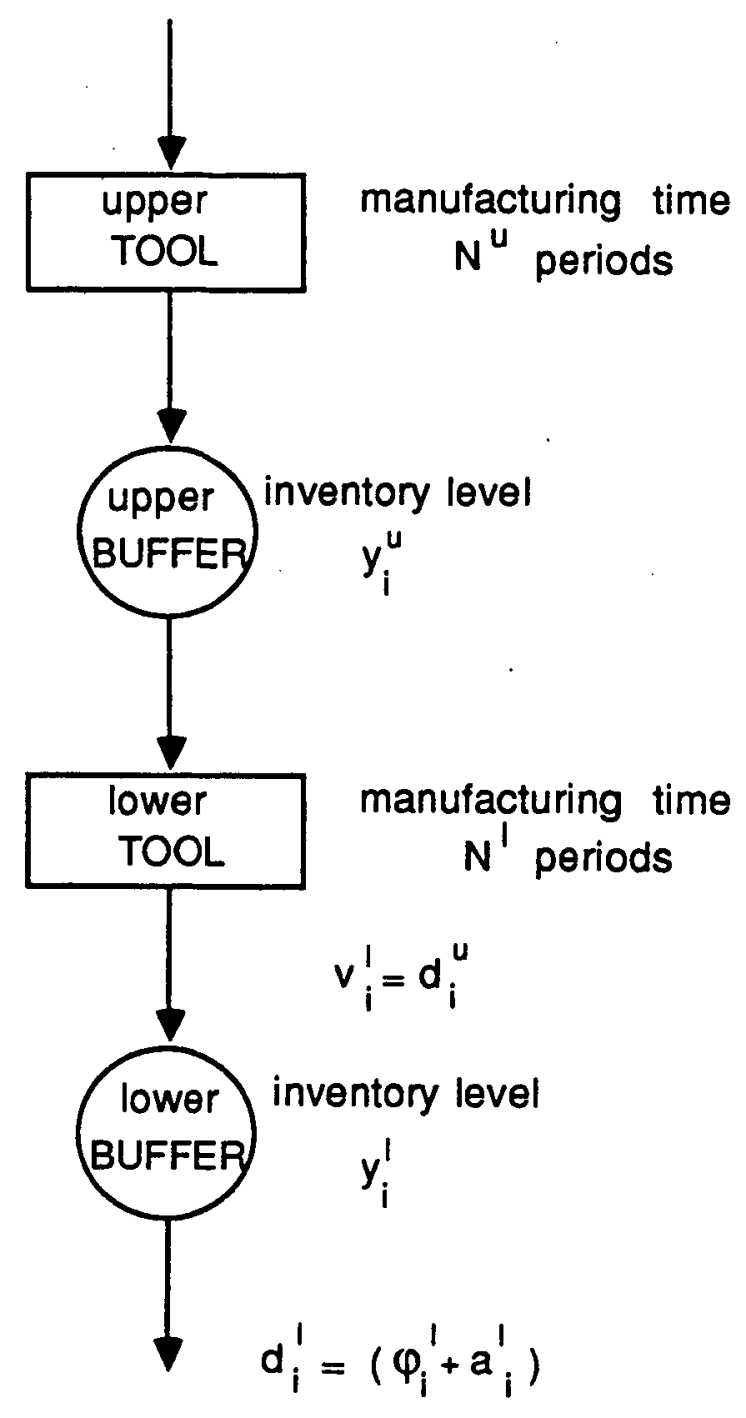

figure 2

As these formulae show it, the first replenishment is deterministic : once the time origin is chosen and the initial inventory level $y_{0}{ }^{1}$ measured, $v_{1}{ }^{1}$ is determined; on the contrary, the next replenishments are not determined until the period when they are to be fixed but we observe that, when they are, they will express as the sum of a term depending only on the estimates and a term that is the value taken by a random normal variable.

That means they are random normal variables as the demand ! 
It is then possible to build the demand estimates for the upper part of the system, as soon as a time origin is chosen :

$$
\varphi_{1}^{u}=v_{1}^{\prime}=\Sigma_{1}^{\prime} F^{-1}\left(1-\varepsilon^{\prime}\right)+\sum_{i=1}^{1+N^{\prime}} \varphi_{i}^{\prime}-\sum_{i=1-N^{\prime}}^{0} v_{i}^{\prime^{*}}-y_{0}^{\prime}
$$

$$
\sigma_{1}^{\mu}=0
$$

and

$\forall k \geq 2 \quad \varphi_{k}^{u}=F^{-1}\left(1-\varepsilon^{\prime}\right)\left(\Sigma_{k}^{\prime}-\Sigma_{k-1}^{\prime}\right)+\varphi_{k+N}^{\prime}+a_{k-1}^{\prime} \quad \sigma_{k}^{u}=\sigma_{k-1}^{\prime}$

If the upper system is assigned the control parameter $\varepsilon^{u}$, then the replenishments will be calculated exactly the same as for the single kernel system :

$$
\begin{aligned}
v_{1}^{u} & =\Sigma_{1}^{u} F^{-1}\left(1-\varepsilon^{u}\right)+\sum_{i=1}^{1+N^{u}} \varphi_{i}^{u}-\sum_{i=1-N^{u}}^{0} v_{i}^{u}-y_{0}^{u} \\
& =\left\{\Sigma_{1}^{u} F^{-1}\left(1-\varepsilon^{u}\right)-\sum_{i=1-N^{u}}^{0} v_{i}^{u}-y_{0}^{u}\right\}+\left\{\sum_{1+N^{u}}^{1} F^{-1}\left(1-\varepsilon^{\prime}\right)-\sum_{i=1-N^{1}}^{0} v_{i}^{1}-y_{0}^{1}\right\}+\sum_{i=1}^{1+N^{u}+N^{\prime}} \varphi_{i}^{\prime}
\end{aligned}
$$

$\forall k \geq 2 \quad v_{k}^{u}=F^{-1}\left(1-\varepsilon^{u}\right)\left(\Sigma_{k}^{u}-\Sigma_{k-1}^{u}\right)+\varphi_{k+N}^{u}+a_{k-1}^{u}$

$$
=F^{-1}\left(1-\varepsilon^{y}\right)\left(\Sigma_{k}^{u}-\Sigma_{k-1}^{u}\right)+F^{-1}\left(1-\varepsilon^{\prime}\right)\left(\Sigma_{k+N}^{\prime} u-\Sigma_{k+N^{\prime}-1}^{\prime}\right)+\varphi_{k+N^{\prime}+N^{\prime}}^{\prime}+a_{k-2}^{\prime}
$$

$$
\text { where } \Sigma_{k}^{u}=\sqrt{\sum_{i=k}^{k+N^{u}} \sigma_{i}^{u^{2}}}
$$

According to the values given to the replenishments, the inventory levels will be defined by the formulae :

$$
\begin{aligned}
& \forall k \geq 1 \quad y_{k+N}^{\prime}=\sum_{k}^{\prime} F^{-1}\left(1-\varepsilon^{\prime}\right)-\sum_{i=k}^{k+N^{\prime}} a_{i}^{\prime} \\
& \text { and } \quad y_{k+N}^{u} u=\sum_{k}^{u} F^{-1}\left(1-\varepsilon^{y}\right)-\sum_{i=k}^{k+N^{u}} a_{i}^{u}=\sum_{k}^{u} F^{-1}\left(1-\varepsilon^{y}\right)-\sum_{i=k-1}^{k+N^{u}-1} a_{i}^{\prime}
\end{aligned}
$$




\section{2/ DETERMINATION OF THE CONTROL PARAMETERS :}

We could have pointed out in the previous paragraph that the absolute probability of going out of stock for the finished product was kept under an $\varepsilon$ different from the control parameters $\varepsilon^{u}$ and $\varepsilon^{\prime}$.In fact, in the kernel model, the replenishment capacity was supposed to be infinite; in the two levels system, this hypothesis can be (and is ) kept for the upper level but not for the lower one : the lower part replenishment at the beginning of a given period must be less than the inventory level reached at the end of previous period by the upper part of the system. Hence the minimal value for the absolute probability of having enough finished product to meet the demand is equal to the product of the minimal relative probability $1-\varepsilon^{\prime}$ of not going out of stock at the lower level (i.e. he minimal probability, provided that the replenishments decided should not be limited by the intermediate inventory level ) by the minimal absolute probability $1-\varepsilon^{u}$ of not going out of stock at the upper level (that is, the probability for the lower-level replenishments not to be limited). product :

Hence the maximal absolute probability of going out of stock for the finished

$$
\varepsilon=\varepsilon^{u}+\varepsilon^{\prime}-\varepsilon^{u} \cdot \varepsilon^{\prime}
$$

This parameter $\varepsilon$ appears in fact as a constraint for the management ; on the contrary, $\varepsilon^{u}$ and $\varepsilon^{\prime}$ will have to be chosen by the production manager. We noticed in the previous paragraph that the statistic value of the inventory depended only on the estimates $(\varphi$ and $\sigma$ ) and the parameter $\varepsilon$. It is then easy to link the statistic mean value of the inventory levels $y^{u}$ and $y^{\prime}$ with the parameters $\varepsilon^{u}$ and $\varepsilon^{\prime}$, in order to compute $\varepsilon^{u}$ and $\varepsilon^{l}$ so as to minimize the total cost of the inventory.

The statistic mean value of the inventory is, in fact, for each level and for the periods that the management can influence :

$$
\begin{aligned}
& \bar{y}^{\prime}=\frac{1}{h-N^{\prime}} \sum_{k=1}^{h-N^{\prime}}\left\{\sum_{k}^{\prime} F^{-1}\left(1-\varepsilon^{\prime}\right)\right\}=F^{-1}\left(1-\varepsilon^{\prime}\right) \frac{\sum_{k=1}^{h-N^{\prime}} \sum_{k}^{\prime}}{h-N^{\prime}}=\alpha^{\prime} F^{-1}\left(1-\varepsilon^{\prime}\right) \\
& \bar{y}^{u}=\frac{1}{h-N^{\prime}-N^{u}} \sum_{k=1}^{h-N^{\prime}-N^{u}}\left\{\sum_{k}^{u} F^{-1}\left(1-\varepsilon^{y}\right)\right\}=F^{-1}\left(1-\varepsilon^{y}\right) \frac{\sum_{k=1}^{h-N^{\prime}-N^{u}} \sum_{k}^{u}}{h-N^{\prime}-N^{u}}=\alpha^{u} F^{-1}\left(1-\varepsilon^{u}\right)
\end{aligned}
$$

If we assume that the costs are linear, which means in particular we take only the variable costs into account, then the statistic mean value of the inventory global cost will express as :

$$
\begin{aligned}
\bar{K}\left(1-\varepsilon^{u}, 1-\varepsilon^{l}\right) & =k^{u} \alpha^{u} F^{-1}\left(1-\varepsilon^{u}\right)+K^{l} \alpha^{l} F^{-1}\left(1-\varepsilon^{\prime}\right) \\
& =K^{u} F^{-1}\left(1-\varepsilon^{u}\right)+K^{\prime} F^{-1}\left(1-\varepsilon^{\prime}\right)
\end{aligned}
$$


We shall then have to solve the problem :

$$
\begin{aligned}
& \operatorname{Min}_{x \in] 0,1[} \bar{K}(x, y) \\
& y \in] 0,1[ \\
& \text { subject to } \quad x x y=1-\varepsilon
\end{aligned}
$$

where $\varepsilon$ is a given parameter .

The point is that neither $F$ nor $F^{-1}$ have explicit formulations : the only formulation for $F$ is :

$$
\dot{F}(u)=\int_{-\infty}^{u} f(t) d t \quad \text { where } f(t)=(1 / \sqrt{2 \cdot \pi}) \cdot \exp \left(-t^{2} / 2\right)
$$

And so we shall have to find the solutions with numerical methods.

First of all, we can notice that the constraint implies that $x>1-\varepsilon$ and $y>1-\varepsilon$; the optimization interval can then be reduced to ] $1-\varepsilon, 1[$. In the following lines, we shall assume that the values given to $\varepsilon$ do not exceed 0.5 (which means that the management will not tolerate that more than fifty percent of the demand could not be met ). In that case, the global cost will be expressed as a convex function of the variable $x$ :

$$
\text { in fact, } \quad\{x . y=1-\varepsilon\} \Leftrightarrow\{y=\alpha / x\} \text { where } \alpha=1-\varepsilon \text {, }
$$

and we can write :

$$
\left.K=K(x)=K^{u} \cdot F^{-1}(x)+K^{\prime} \cdot F^{-1}(\alpha / x) \text { with } x \in\right] \alpha, 1[
$$

$F$ is concave on the interval $\left[0,+\infty\left[\left(F^{\prime \prime}(t)<0\right)\right.\right.$ and takes values ranging from 0.5 to 1 . Then $\mathrm{F}^{-1}$ is convex on the interval $\left[0.5,1\right.$ [ and, as $\mathrm{dF}^{-1} / \mathrm{dx}$ is positive on this interval, $\mathrm{K}$ is convex on [ $0.5,1$ [ .

It is then always possible to find a solution to the problem previously posed, for example by means of a gradient method: there is only one value for the unknown $x$ that minimizes the cost $K$.

The graphs presented hereafter show the evolution of the global inventory cost as a function of $X=1-\varepsilon^{1}$ for different values of parameter $K^{u}$ - parameter $K^{\prime}$ remaining constant and equal to 100 - and for different values of parameter $\varepsilon$ which is, in fact, a measure of the reliability of the system facing the demands.

These graphs demonstrate that :

$1 /$ The more demanding the system becomes in terms of service to the client, the higher global inventory cost (the curves do not cross one another).

2/ The optima' control is stricter for the cheaper stock and hence imposes a higher safety stock at that level. 

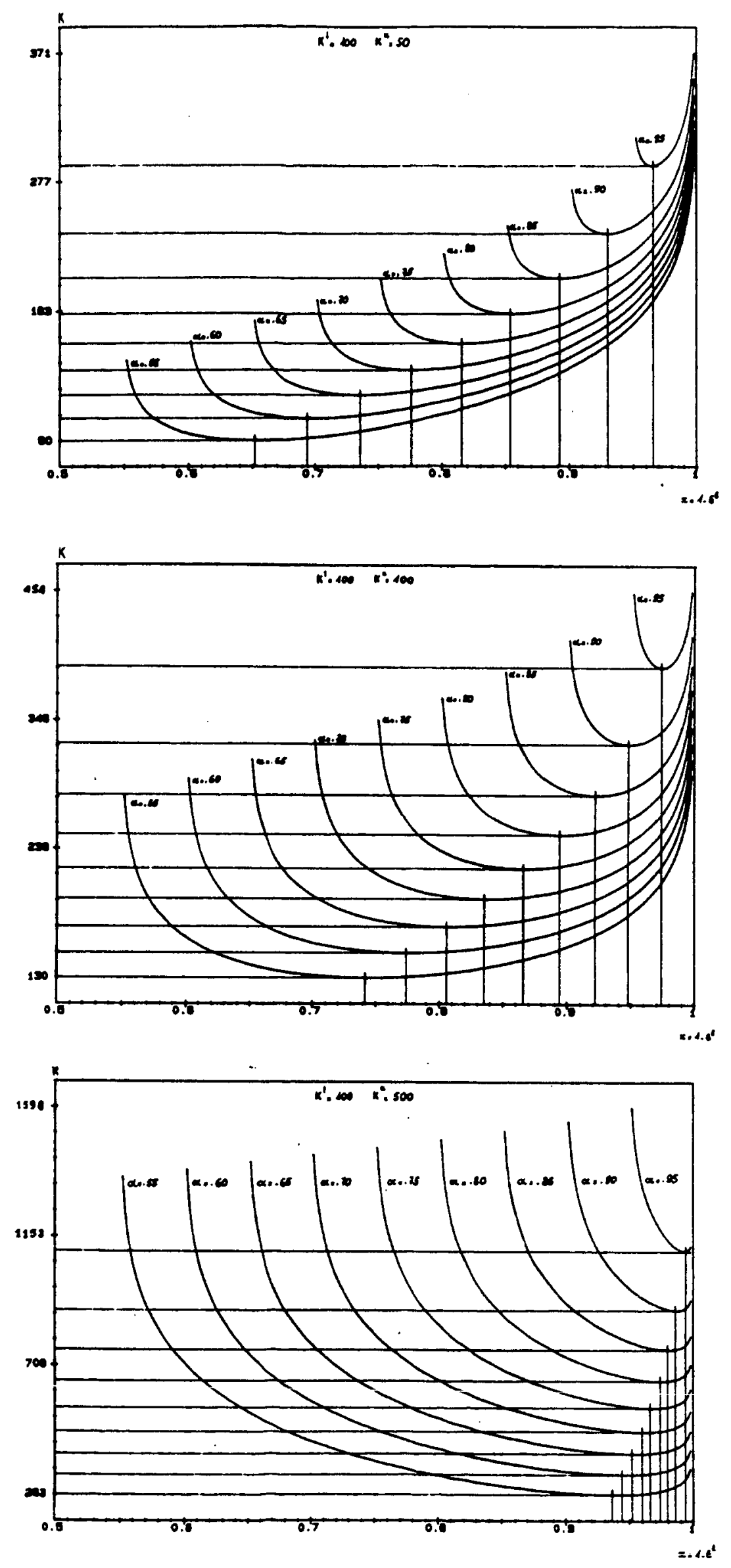


\section{CONCLUSION :}

This paper did not present the most general formulae developped to deal with the replenishment control of any flow-shop; it is however obvious that the generalization is easy. The main further research that can be thought of concerns the introduction of finite capacities : the policy previously described has already been adapted to a finite capacity model in the case of the kernel system; this approach introduces a new control parameter to bound the probability for the future minimal replenishments given by our formula to exceed the production capacity or lead to an overstock. The replenishment actually decided is either equal to its theoretical value if this one is less than the the capacity or equal to the capacity ; then it cannot rightfully be considered as a random normal variable and the genelazation to mutiple level systems requires a valuation of the bias introduced.

\section{REFERENCES}

Coillard, P. and J.M. Proth (1983) "Sur I' Effet des Stocks Tampons dans une Fabrication en Ligne".

Giard, V. rapport n ${ }^{\circ} 5$ du projet LAPON à la Fondation Nationale pour l' Enseignement de la Gestion en Entreprises.

Kimemia, J. G. (1982) "Hierarchical Control of Production in Flexible Manufacturing Systems" , Massachusetts Institute of Technology Laboratory for Information and Decision Systems . Report LIDS - TH - 1215

Akella, R., Y. Choong, and S.B. Gershwin (1984) "Performance of Hierarchical Production Scheduling Policy ", Massachusetts Institute of Technology Laboratory for Information and Decision Systems . Report LIDS - FR - 1357

Bensoussan, A. and J.M.Proth (1982) "Inventory planning in a deterministic environment : Continuous time models with concave costs ". European Journal of Operational Research.

Hildebrandt, R. and R. Suri "Modelling Flexible Manufacturing Systems Using Mean Value Analysis". 
